\title{
The Effect of Electroencephalography Leads on Image Quality in Cerebral Perfusion SPECT and ${ }^{18}$ F-FDG PET/CT
}

\author{
Lulu Zhang ${ }^{1}$, Stephanie P. Yen ${ }^{1}$, Marc A. Seltzer ${ }^{1}$, George P. Thomas, Jr. $^{2}$, Kristen Willis ${ }^{1}$, and Alan Siegel ${ }^{1}$ \\ ${ }^{1}$ Department of Radiology, Dartmouth-Hitchcock Medical Center, Lebanon, New Hampshire; and ${ }^{2}$ Department of Neurology, \\ Dartmouth-Hitchcock Medical Center, Lebanon, New Hampshire
}

Cerebral perfusion SPECT and ${ }^{18}$ F-FDG PET/CT are commonly performed diagnostic procedures for patients with epilepsy. Individuals undergoing these tests are often inpatients with electroencephalography leads. We have routinely removed these leads because of concern that they would lead to imaging artifacts. The leads would then be replaced at the conclusion of the scan. The goal of our study was to determine whether the electroencephalography leads actually do cause artifacts that can lead to erroneous scan interpretation or make the scan uninterpretable. Methods: ${ }^{18} \mathrm{~F}-\mathrm{FDG} \mathrm{PET} / \mathrm{CT}$ and ${ }^{99 \mathrm{~m} T \mathrm{Tc}-\text { pertechnetate }}$ SPECT were performed on a 2-dimensional brain phantom. The phantom was scanned with standard leads, CT/MR-compatible leads, and no leads. The quality of the images was ranked by 3 experienced nuclear medicine physicians, who then determined whether they could differentiate each of the scans from a scan in which it was known that no leads were present. Results: No differences could be detected between scans obtained without leads and scans obtained with either set of leads. The standard electroencephalography leads did create artifacts in the CT portion of the PET/CT images, whereas the CT/MR-compatible leads did not. Conclusion: This phantom study suggests that electroencephalography leads, whether standard or CT/MRcompatible, do not need to be removed for SPECT or PET procedures. Further study evaluating the effect on actual patient scans would be of value to support this conclusion.

Key Words: EEG leads; cerebral perfusion SPECT; ${ }^{18} \mathrm{~F}-\mathrm{FDG}$ PET/CT; brain phantom

J Nucl Med Technol 2018; 46:359-361

DOI: 10.2967/jnmt.117.207621

\section{$\mathbf{E}$} pilepsy is characterized by an enduring predisposition for seizures due to abnormal excessive or synchronous neuronal activity in the brain (1). In most cases, the seizures can be controlled with medication, but approximately $30 \%$ of patients have inadequate control despite maximal medical therapy (2). Some of these patients will have epileptogenic foci that may be amenable to surgical resection.

\footnotetext{
Received Jan. 2, 2018; revision accepted May 2, 2018.

For correspondence or reprints contact: Alan Siegel, Department of Radiology, Dartmouth-Hitchcock Medical Center, One Medical Center Dr., Lebanon, $\mathrm{NH} 03756$.

E-mail: alan.h.siegel@hitchcock.org

Published online Jun. 8, 2018.

COPYRIGHT @ 2018 by the Society of Nuclear Medicine and Molecular Imaging
}

Approximately $60 \%-70 \%$ of patients who undergo surgical resection have long-term improvement or become seizure-free $(3,4)$. Thus, in patients with medically intractable epilepsy, the identification of an isolated epileptogenic focus can greatly improve the patient's quality of life.

A multimodality approach is frequently required to identify and map the extent of lesions. MRI is excellent for identifying seizure-related structural abnormalities such as mesial temporal sclerosis or focal cortical dysplasia $(5,6)$. Functional neuroimaging modalities such as SPECT and ${ }^{18}$ F-FDG PET/CT can identify morphologically inconspicuous or structurally normal epileptogenic areas and thus improve overall sensitivity and specificity for detecting isolated seizure foci (7).

Interictal evaluation is important in the identification of epileptogenic foci using both SPECT and PET/CT. Brain perfusion scans with SPECT for epilepsy rely on a relatively increased flow to the epileptogenic foci during the seizure. The sensitivity of the examination for detecting an area of increased perfusion is greater if interictal scans are subtracted from ictal scans (8). The pharmacokinetics and logistic complications of using ${ }^{18} \mathrm{~F}$-FDG limit use of ictal PET scans in routine clinical practice. Rather, PET relies on the relative hypometabolism of the epileptogenic foci in the interictal period for localization. Thus, for both modalities, it is vital that the patient not be undergoing a clinically silent seizure during the interictal scan. This is ensured with electroencephalography evaluation just before and after the interictal scan. At our institution, the electroencephalography leads are routinely removed and then replaced for each scan because of concern that the leads may create artifacts. In the SPECT or PET examination, the leads themselves may lead to photon attenuation. In addition, in the PET examination the streak artifacts on the CT images may propagate to the attenuation-corrected nonfused PET images, thus leading to artifactual defects. However, the process of removing and replacing electroencephalography leads requires coordination between at least 2 departments and thus consumes human resources and can cause significant delays in workflow. In this phantom study, we investigated the effect of standard and MRI/CT-compatible electroencephalography leads on PET and SPECT images. 


\section{MATERIALS AND METHODS}

A Hoffman 2-dimensional brain phantom (Data Spectrum) was used for both SPECT and PET/CT scans (Fig. 1). For SPECT (Symbia T6; Siemens), $185 \mathrm{MBq}(5 \mathrm{mCi})$ of ${ }^{99 \mathrm{~m}} \mathrm{Tc}$-pertechnetate were used, and 15-min scans were performed consecutively without electroencephalography leads, with MRI/CT-compatible leads (Ives Conductive Plastic Electrodes CPE 25/d; Natus), and with standard electroencephalography leads (Genuine Grass Gold Disk Electrodes; Natus). A noncircular orbit with 32 views (18 per head) at $30 \mathrm{~s}$ per view and a continuous acquisition with a $128 \times$ 128 matrix were used. SPECT images were reconstructed using a Chang attenuation correction technique.

For PET (Discovery ST; GE Healthcare), $185 \mathrm{MBq}(5 \mathrm{mCi})$ of ${ }^{18}$ F-FDG were used with 8 -min acquisitions, a $512 \times 512$ matrix, and the same types of leads. There were 10 leads positioned on the surface of the phantom. The positioning was the same for both the standard and the CT/MRI-compatible leads. Each set of leads was scanned once for SPECT and once for PET/CT. CT-based attenuation correction was performed for the PET scans. The CT scan for attenuation correction was acquired for $10 \mathrm{~s}$ with $120 \mathrm{kV}$ and 60 mAs.

The SPECT images obtained with and without the 2 different types of electroencephalography leads were randomized and presented to 3 experienced nuclear medicine physicians independently on a PACS (iSite; Philips) workstation. The readers had an average of $21 \mathrm{y}$ of evaluating SPECT and PET/CT images for epilepsy. The same was then done for the attenuation-corrected PET scans. For both the PET and the SPECT images, each reader was asked to rank, from best to worst, the quality of the images for each of the 3 scans. The readers were then given each of the 3 image sets masked to the presence of leads and were asked to compare them with the image set known to have no leads. Finally, the readers were asked to rank the quality of images, from best to worst, from the CT portion of the PET examinations (Fig. 2).

\section{RESULTS}

None of the readers could detect any differences among studies with no leads, with MRI/CT-compatible leads, and with standard leads for either the SPECT or the PET

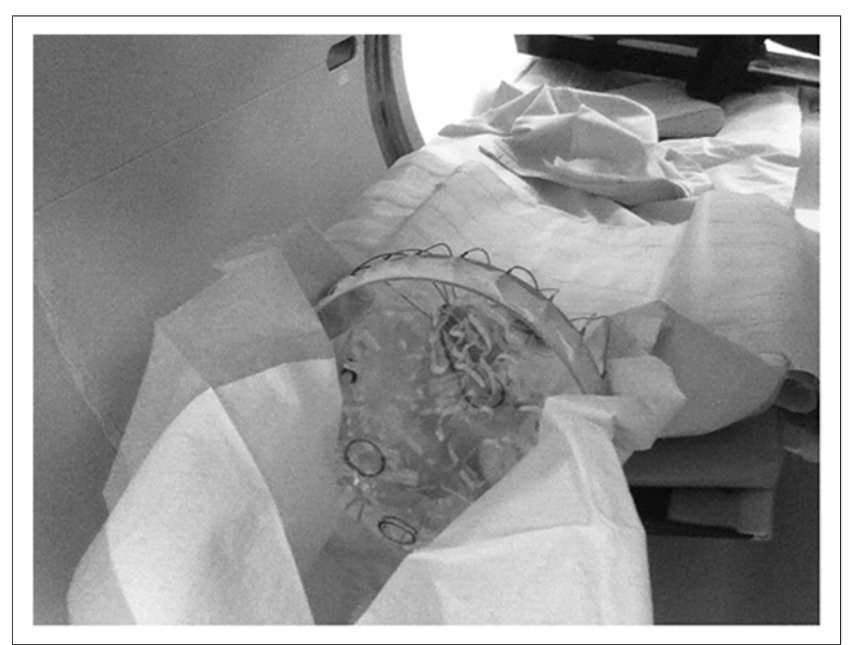

FIGURE 1. Photograph of 2-dimensional brain phantom demonstrating configuration of surface leads.

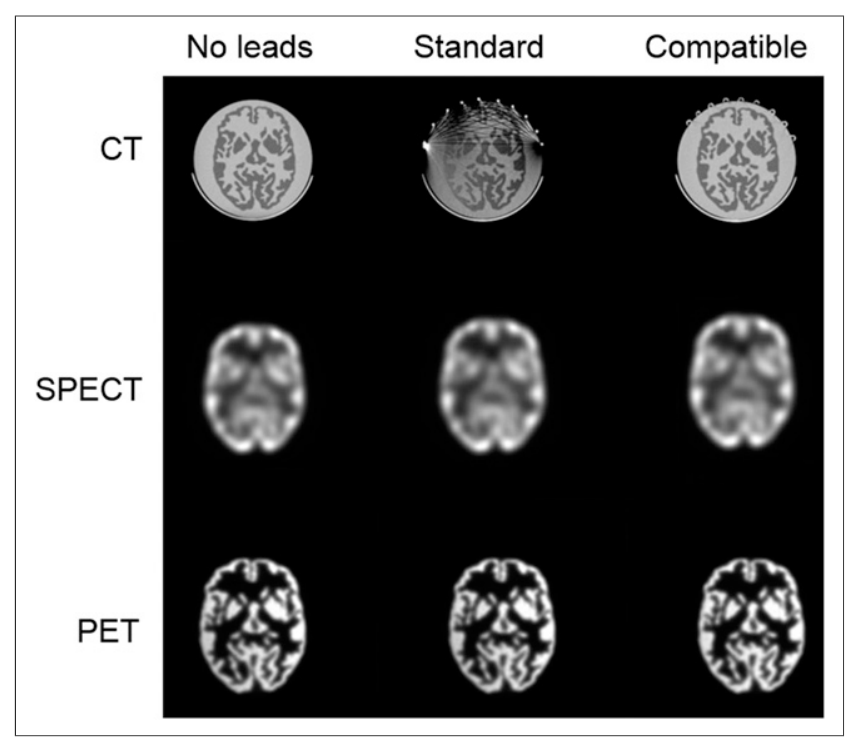

FIGURE 2. Scans of brain phantom: CT images (as a component of PET/CT), SPECT images, and PET images, each without leads, with standard electroencephalography leads, and with MRI/CT-compatible leads.

examinations. Even when given a scan with no leads as a control, all 3 readers still saw no perceptible differences. All SPECT and all PET images were ranked as equivalent. For the CT portion of the examinations, all 3 readers identified the standard-electroencephalography-lead scans as degraded in quality. The scans displayed streak artifacts that limited visualization of a portion of the brain and could seriously hinder the interpretation. All 3 readers also identified the presence of MRI/CT-compatible leads on the surface of the phantom on the CT images but saw no differences in the image quality of the simulated intracranial structures between the scan with MRI/CT-compatible leads and the scan with no leads.

\section{DISCUSSION}

Functional nuclear imaging plays an important role in the identification of surgically resectable epileptogenic foci for medically refractory epilepsy. Both cerebral perfusion imaging with SPECT and glucose metabolism imaging with PET require accurate interictal imaging confirmed by electroencephalography monitoring. Metallic leads are known to cause significant streak artifacts on CT images, raising concern about a decrease in diagnostic accuracy because of the artifacts in the SPECT or PET images. If artifacts occur, the impact on scan interpretation may be significant, especially if the affected area is that of clinical concern. Our study showed that although regular electroencephalography leads cause artifacts on CT images, these leads do not result in perceptible degradation of the SPECT or PET images. All 3 experienced nuclear medicine physicians saw no differences in image quality among examinations with standard electroencephalography leads, with MRI/ CT-compatible leads, and without leads. A prior report by 
Lemmens et al. found that the measurable attenuation artifacts from metallic electroencephalography leads could be sufficiently reduced by metallic artifact reconstruction algorithms to not affect visual assessment of the study (9).

Our findings indicate that the presence of electroencephalography leads does not affect the quality of images in studies with SPECT or ${ }^{18}$ F-FDG PET. Standard electroencephalography leads do create artifacts within the CT images. The CT/MRI-compatible leads have no visible effect on the SPECT or PET images and do not create artifacts in the CT images.

We do recognize the limitation of performing this analysis on a phantom study. It would be valuable to further test our hypothesis in the clinical setting using both qualitative and quantitative methods. For 3-dimensional images of the brain, it may be worthwhile to add image registration, normalization, and subtraction analysis. This technique has been shown to improve detection of subtle changes between image sets $(10,11)$.

\section{CONCLUSION}

The results of our phantom study suggest that the presence of electroencephalography leads, whether standard or MRI/CT-compatible, does not lead to a clinically relevant degradation of the quality of the SPECT or PET images and that it may not be necessary to have the leads removed during scan acquisition. Leaving the leads in place would lead to shorter times for study preparation, increased patient comfort, more efficient use of resources, and thus potential savings in cost.

\section{DISCLOSURE}

No potential conflict of interest relevant to this article was reported.

\section{REFERENCES}

1. Fisher RS, Acevedo C, Arzimanoglou A, et al. ILAE official report: a practical clinical definition of epilepsy. Epilepsia. 2014;55:475-482.

2. Engel J. Introduction to temporal lobe epilepsy. Epilepsy Res. 1996;26:141-150.

3. Cohen-Gadol AA, Ozduman K, Bronen RA, Kim JH, Spencer DD. Long-term outcome after epilepsy surgery for focal cortical dysplasia. J Neurosurg. 2004; 101:55-65.

4. Wiebe S, Blume WT, Girvin JP, Eliasziw M. A randomized, controlled trial of surgery for temporal-lobe epilepsy. N Engl J Med. 2001;345:311-318.

5. Cross JH, Jackson GD, Neville BG, et al. Early detection of abnormalities in partial epilepsy using magnetic resonance. Arch Dis Child. 1993;69:104-109.

6. Coan AC, Kubota B, Bergo FPG, Campos BM, Cendes F. 3T MRI quantification of hippocampal volume and signal in mesial temporal lobe epilepsy improves detection of hippocampal sclerosis. AJNR Am J Neuroradiol. 2014;35:77-83.

7. Spencer SS. The relative contributions of MRI, SPECT, and PET imaging in epilepsy. Epilepsia. 1994;35(suppl 6):S72-S89.

8. la Fougère C, Rominger A, Forster S, Geisler J, Bartenstein P. PET and SPECT in epilepsy: a critical review. Epilepsy Behav. 2009;15:50-55.

9. Lemmens C, Montando M, Nuyts J, et al. Impact of metal artefacts due to EEG electrodes in brain PET/CT imaging. Phys Med Biol. 2008;53:4417-4429.

10. Kaiboriboon K, Lowe VJ, Chantarujikapong SI, Hogan RE. The usefulness of subtraction ictal SPECT coregistered to MRI in single- and dual-headed SPECT cameras in partial epilepsy. Epilepsia. 2002;43:408-414.

11. Lewis PJ, Siegel A, Siegel AM, et al. Does performing image registration and subtraction in ictal brain SPECT help localize neocortical seizures? J Nucl Med. 2000;41:1619-1626. 